\title{
Myositis-Aktivitätenprofil (MAP)
}

\section{(Fragebogen über tägliche Aktivitäten)}

(adaptiert nach Alexanderson $\mathrm{H}$ et al. The myositis activity profile - Initial validation for assessment of polymyositis/dermatomyositis in the USAR. J Rheumatol 2012;39:11; Alexanderson $\mathrm{H}$ et al. Development of the myositis activities profile - validity and reliability of a self-administered questionnaire to assess activity limitations in patients with polymyositis/dermatomyositis. J Rheumatol 2002;29:11) 
Bitte beantworten Sie die folgenden Fragen über Ihre tägliche Aktivitäten. Kreuzen sie das Feld an, bei dem Sie das Gefühl haben, dass es Ihre Erfahrungen der letzten sieben Tagen am besten beschreibt.

„Schwierigkeiten“ beinhaltet dabei sowohl wie anstrenged es für Sie war, eine Aktivität auszuüben, als auch wie wichtig es für Sie war, die beschriebene Aktivität ausführen zu können. Versuchen Sie Ihre Schwierigkeiten mit Ihren Bedürfnissen zu gewichten, wenn Sie die Fragen beantworten.

Beispiel 1: Sie haben grosse Mühe in die Hocke zu gehen (und Aktivitäten auszuführen), aber Sie mussten dies in der letzten Woche nicht tun. Daher können Sie diese Aktivität als wenig schwierig einstufen.

Beispiel 2: Sie haben geringe Mühe beim Kochen, jedoch mussten/wollten Sie in der letzten Woche mehrmals täglich kochen. Daher können Sie diese Aktiviät als sehr schwierig einstufen.

Beantworten Sie nun bitte alle folgenden Fragen mit einer bestmöglichen Einschätzung und kreuzen Sie jeweils nur ein Feld pro Frage an. 


\section{Bewegung}

1) Wie schwierig ist es für Sie in Ihrem Alltag während längerer Zeit zu stehen (z.B. in einer Schlange anzustehen)?
(1)
(2)
(3)
(4)
$\square$

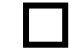
Gar nicht sehr wenig schwierig schwierig

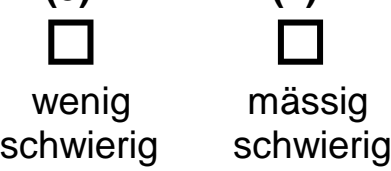
(5)

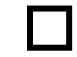
sehr schwierig
(6)

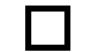
(7)
extrem schwierig

2) Wie schwierig ist es für Sie in Ihrem Alltag beim Vordersitz eines Autos ein bzw- auszusteigen?
(1)
(2)
(3)
(4)
(5)
(6)

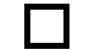
Gar nicht sehr wenig schwierig schwierig

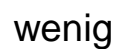
schwierig

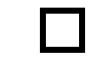
mässig schwierig

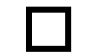
sehr schwierig

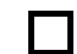
extrem schwierig
(7)

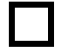
unmöglich

3) Wie schwierig ist es für Sie in Ihrem Alltag einen Gegenstand vom Boden aufzuheben (zum Beispiel etwas vom Boden auf den Tisch zu heben)?
(1)
(2)
(3)
(4)
(5)
(6)
(7)
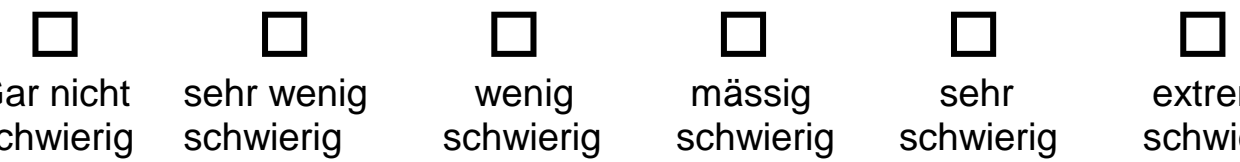

4) Wie schwierig ist es für Sie in Ihrem Alltag eine Umhängetasche oder einen Rucksack mit mehr als einem Kilo Gewicht zu tragen?
(1)
(2)
(3)
(4)
(5)
(6)
(7)

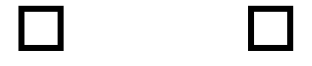
Gar nicht sehr wenig
wenig
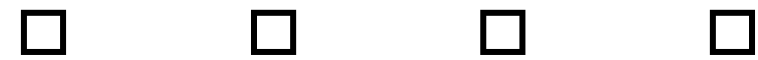
schwierig schwierig
schwierig
mässig
sehr
schwierig
extrem schwierig
unmöglich

5) Wie schwierig ist es für Sie in Ihrem Alltag eine volle Einkaufstasche vom Ladentisch oder vom Auto auf den Boden zu stellen?
(1)
(2)
(3)
(4)
(5)
(6)
(7)
$\square \quad \square$
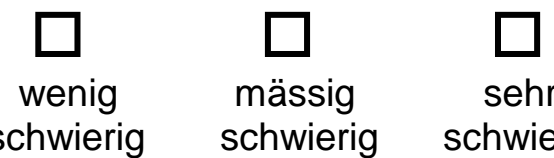
sehr
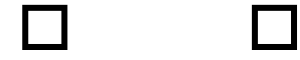
schwierig schwierig
schwierig
schwierig
extrem schwierig
unmöglich

6) Wie schwierig ist es für Sie in Ihrem Altag ein Kind, Haustier oder Gegenstände in ihre Armen hoch zu heben?
(1)
(2)
(3)
(4)
(5)
(6)
(7)
Gar nicht sehr wenig

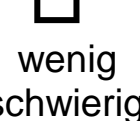
mässig
$\square$

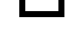
extrem schwierig
unmöglich
schwierig schwierig schwierig
schwierig

$$
\text { schwierig }
$$


7) Wie schwierig ist es für Sie in Ihrem Alltag Gegenstände mit einer oder beiden Händen zu greifen (beispielsweise eine Bratpfanne oder einen Hammer)?
(1)
(2)
(3)
(4)
(5)
(6)
(7)
Gar nicht
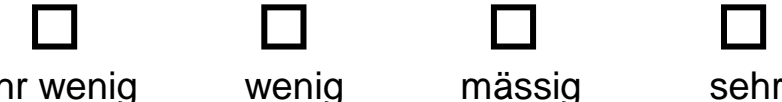
schwierig schwierig
schwierig
schwierig schwierig
extrem schwierig

8) Wie schwierig ist es für Sie in Ihrem Alltag Konfitürengläser, Frischhaltedosen

(z.B. Tupperware) oder Verpackungen zu öffnen?
(1)
(2)
(3)
(4)
(5)
(6)
(7)
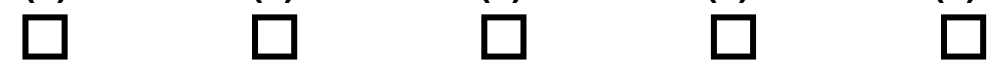
Gar nicht sehr wenig schwierig schwierig
wenig mässig sehr schwierig
schwierig
schwierig
extrem schwierig

9) Wie schwierig ist es für sie für Sie in Ihrem Alltag eine schwere Türe zu öffnen (beispielsweise eine Auto-, Restaurant-, oder Ladentüre)?

(1)

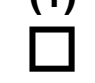

Gar nicht schwierig
(2)

sehr wenig schwierig
(3)

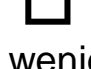
schwierig
(4)

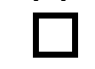

mässig schwierig
(5)

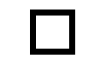

sehr schwierig
(6)

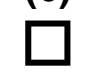

extrem schwierig
(7)

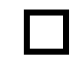

unmöglich

\section{Aktivitäten der Fortbewegung}

10) Wie schwierig ist es für Sie in Ihrem Alltag mehr als 800 Meter auf einem ebenen Boden zu gehen?
(1)
(2)
(3)
(4)
(5)
(6)
(7)
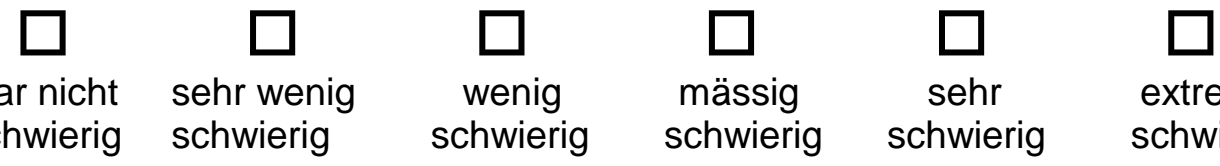
extrem schwierig

Gar nicht

11) Wie schwierig ist es für Sie in Ihrem Alltag zu rennen (beispielsweise auf einen Bus zu rennen oder zu joggen)?
(1)
(2)
(3)
(4)
(5)
(6)
(7)
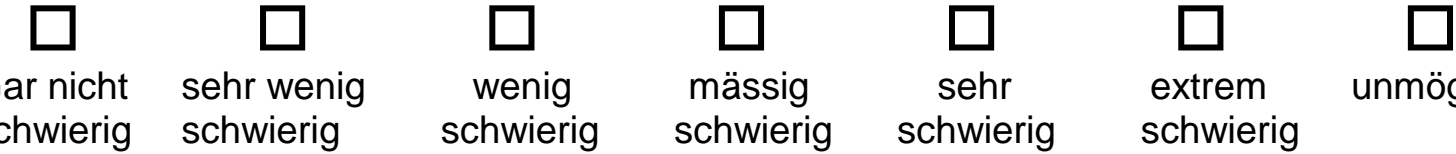
sehr
extrem schwierig schwierig schwierig
unmöglich

12) Wie schwierig ist es für Sie in Ihrem Alltag ein Stockwerk Treppe ohne Geländer zu steigen?
(1)
(2)
(3)
(4)
(5)
(6)
(7)
$\square \quad \square$
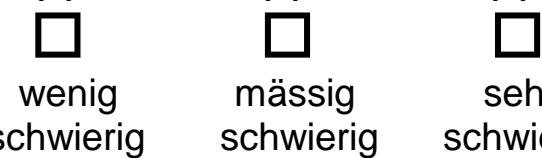
sehr schwierig

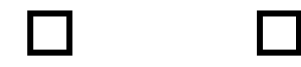
extrem unmöglich schwierig 
13) Wie schwierig ist es für Sie in Ihrem Alltag öffentliche Verkehrsmittel wie einen Bus, einen Zug oder ein Flugzeug zu benützen?
(1)
(2)
(3)
(4)
(5)
(6)
(7)
$\square$
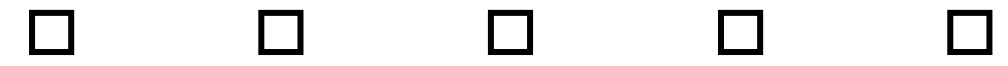
$\square$
schwierig schwierig
wenig mässig
sehr
extrem
unmöglich schwierig schwierig schwierig
schwierig

\section{Körperpflege und -hygiene}

14) Wie schwierig ist es für Sie in Ihrem Alltag Ihre Haare zu waschen?

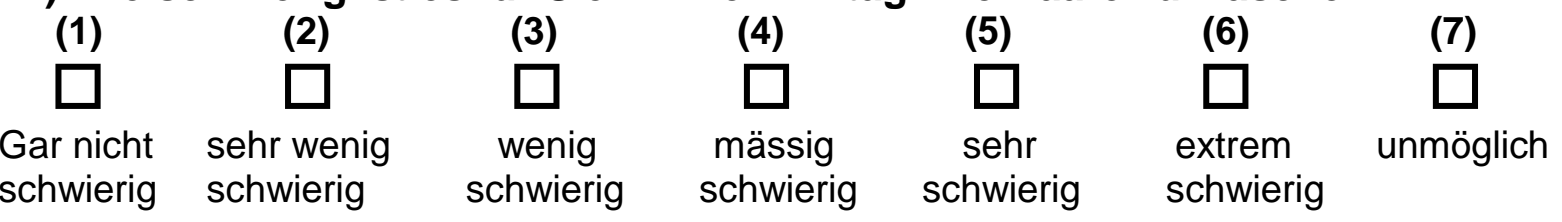

15) Wie schwierig ist es für Sie in Ihrem Alltag ein Bad in einer Badewanne zu nehmen?
(1)
(2)
(3)
(4)
(5)
$\square$
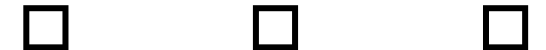
(6)
(7)
Gar nicht sehr wenig schwierig schwierig
wenig schwierig
mässig schwierig
sehr schwierig

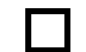
extrem schwierig

16) Wie schwierig ist es für Sie in Ihrem Altag Ihre Haare zu kämmen oder zu föhnen?
(1)
(2)
(3)
(4)
(5)
(6)
(7)

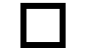
Gar nicht sehr wenig schwierig schwierig

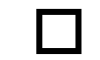
wenig schwierig

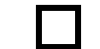
mässig schwierig

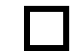
sehr schwierig

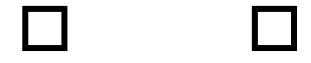
extrem schwierig
unmöglich

17) Wie schwierig ist es für Sie in Ihrem Alltag Ihre Zehennägel zu schneiden oder zu feilen?
(1)
(2)
(3)
(4)
(5)
(6)
(7)

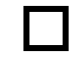
Gar nicht sehr wenig schwierig schwierig
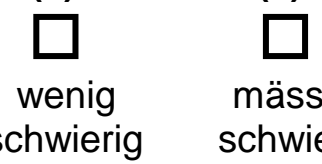
mässig schwierig

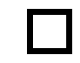
sehr schwierig

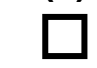
extrem schwierig

18) Wie schwierig ist es für Sie in Ihrem Altag Ihren Rücken zu waschen (eventuell mit einer Rückenbürste)?
(1)
(2)
(3)
(4)
(5)
(6)
(7)
$\square \quad \square$
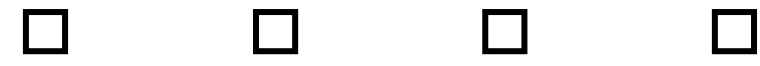
schwierig schwierig
wenig
mässig
sehr
extrem schwierig
unmöglich schwierig schwierig schwierig 
19) Wie schwierig ist es für Sie in Ihrem Alltag sich nach dem Gang zur Toilette selbst zu reinigen?
(1)
(2)
(3)
(4)
(5)
(6)
(7)
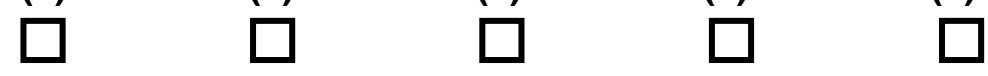
$\square$

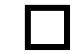
Gar nicht sehr wenig
schwierig schwierig

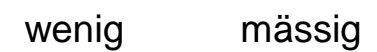
sehr
extrem
unmöglich

20) Wie schwierig ist es für Sie in Ihrem Alltag einen Pullover an- bzw. auszuziehen?
(1)
(2)
(3)
(4)
(5)
(6)
(7)

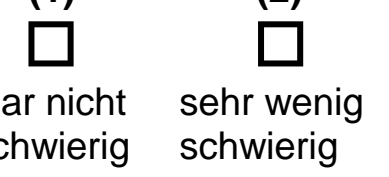
wenig mässig
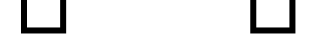
extrem schwierig
unmöglich

21) Wie schwierig ist es für Sie in Ihrem Alltag Hosen oder Strumpfhosen anbzw. auszuziehen?
(1)
(2)
(3)
(4)
(5)
(6)
(7)

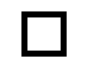
schwierig schwierig

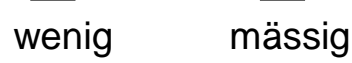
schwierig schwierig

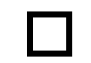
sehr
schwierig

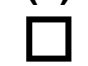
extrem schwierig
unmöglich

Gar nicht

22) Wie schwierig ist es für Sie in Ihrem Alltag Schuhe oder Stiefel an- bzw. auszuziehen?
(1)
(2)
(3)
(4)
(5)
(6)
(7)
Gar nicht sehr wenig

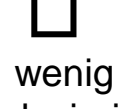
mässig
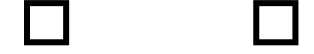
schwierig schwierig schwierig
schwierig
sehr
extrem schwierig
unmöglich

\section{Arbeiten im Haushalt}

23) Wie schwierig ist es für Sie in Ihrem Alltag tägliche Einkäufe nach Hause zu transportieren?
(1)
(2)
(3)
(4)
(5)
(6)
(7)

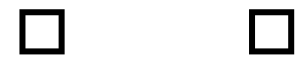
Gar nicht sehr wenig schwierig schwierig
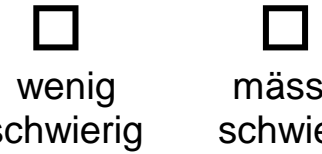
mässig schwierig

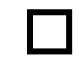
sehr schwierig

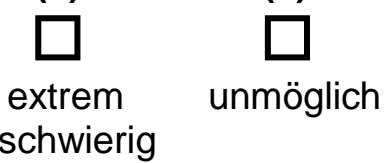

24) Wie schwierig ist es für Sie in Ihrem Alltag Teller oder Gläser in den oberen Teil des Schrankes zu versorgen?
(1)
(2)
(3)
(4)
(5)
(6)
(7)

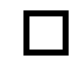
Gar nicht sehr wenig schwierig schwierig

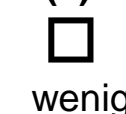
schwierig

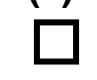
mässig schwierig

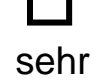 schwierig
extrem schwierig 
25) Wie schwierig ist es für Sie in Ihrem Alltag den Boden nass aufzunehmen?
(1)
(2)
(3)
(4)
(5)
(6)
(7)
Gar nicht
sehr wenig
wenig
mässig
sehr
extrem
unmöglich
schwierig schwierig
schwierig
schwierig
schwierig schwierig

26) Wie schwierig ist es für Sie in Ihrem Alltag zu staubsaugen?
(1)
(2)
(3)
(4)
(5)
(6) (7)
$\begin{array}{ll}\text { Gar nicht } & \text { sehr wenig } \\ \text { schwierig } & \text { schwierig }\end{array}$
wenig mässig

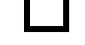
schwierig schwierig
sehr
extrem
unmöglich

27) Wie schwierig ist es für Sie in Ihrem Alltag Ihr Haus und Garten oder Ihre Wohnung in Stand zu halten (beispielsweise Fenster zu putzen oder den Rasen zu mähen)?
(1)
(2)
(3)
(4)
(5)
(6)
(7)

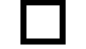
Gar nicht sehr wenig schwierig schwierig

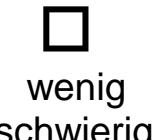
schwierig

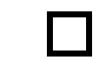
mässig schwierig

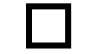
sehr schwierig

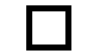
extrem schwierig

28) Wie schwierig ist es für Sie in Ihrem Alltag Haushaltsgeräte zu reinigen und in Stand zu halten (beispielsweise einen Staubsauger, einen Ofen oder elektrische Geräte)?
(1)
(2)
(3)
(4)
(5)

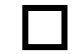
wenig schwierig

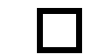
mässig schwierig
(6)

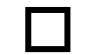
(7)

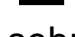
sehr schwierig
extrem schwierig
unmöglich

29) Wie schwierig ist es für Sie in Ihrem Alltag den Kontakt mit Freunden und Verwandten aufrecht zu erhalten bzw. sie zu treffen?
(1)
(2)
(3)
(4)
(5)
(6)

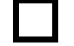
(7)
Gar nicht sehr wenig
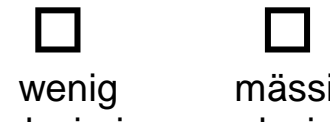
sehr
mässig
schwierig
schwierig
extrem schwierig
unmöglich

30) Wie schwierig ist es für Sie in Ihrem Alltag Überanstrengung zu vermeiden?
(1)
(2)
(3)
(4)
(5)
(6)
(7)
Gar nicht sehr wenig

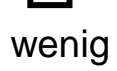
mässig
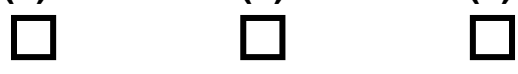
schwierig schwierig
schwierig
schwierig
schwierig
extrem schwierig
unmöglich 
31) Wie schwierig ist es für Sie in Ihrem Altag ihre Arbeit, Schule und/oder Hausarbeit zu bewältigen?
(1)
(2)
(3)
$\square$
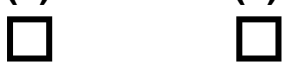
(4)

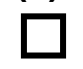
(5)
(6)
(7)
Gar nicht sehr wenig
schwierig schwierig
wenig
mässig

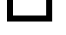
sehr

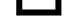
extrem
unmöglich schwierig schwierig schwierig schwierig

32) Wie schwierig ist es für Sie in Ihrem Alltag Freizeitaktivitäten, die Ihnen Spass machen, ausführen zu können?
(1)
(2)
(3)
(4)
(5)
(6)
(7)
$\square$

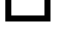
wenig schwierig

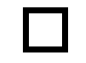
mässig schwierig

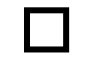
sehr schwierig

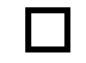
extrem schwierig

Gar nicht

schwierig

\section{Besten Dank für das Ausfüllen des Fragebogens!}




\section{Auswertung des Myositis-Aktivitätenprofils}

Organisieren Sie alle Antworten in jeder Kategorie von 1-7, wobei 1 "überhaupt nicht schwierig" und 7 "unmöglich" ist

Kategorie „Bewegung“ mittlerer Wert $=4$. Wert vom niedrigsten aus

Kategorie „Aktivitäten der Fortbewegung“ mittlerer Wert $=2$. Wert vom niedrigsten aus

Kategorie „Körperpflege und Hygiene“ mittlerer Wert $=5$. Wert vom niedrigsten aus

Kategorie „Arbeiten im Haushalt“ mittlerer Wert $=3$. Wert vom niedrigsten aus

Die vier Einzelfragen über „soziale Aktivitäten“, „Vermeidung von Überanstrengung“, „Arbeit/Schule/Haushalt" und „Erholung“ sind in keiner Kategorie enthalten und werden jeweils separat als Einzelkategorien gewertet.

Schreiben Sie den mittleren Wert sowohl jeder Kategorie als auch jeder Antwort von den Einzelfragen in die untenstehende Tabelle.

Mittlerer Wert = 1 bedeutet “überhaupt nicht schwierig" die Aktivität eines Abschnittes oder einer Einzelfrage auszuführen

Mittlerer Wert = 7 bedeutet "unmöglich" die Aktivität eines Abschnittes oder einer Einzelfrage auszuführen

\begin{tabular}{|c|c|}
\hline \multicolumn{2}{|l|}{ Datum } \\
\hline Subskala/Einzelfrage & $\begin{array}{l}\text { Mittlere Wert / } \\
\text { Median }\end{array}$ \\
\hline \multicolumn{2}{|l|}{ Bewegung } \\
\hline \multicolumn{2}{|l|}{ Aktivitäten der Fortbewegung } \\
\hline \multicolumn{2}{|l|}{ Körperpflege und -hygiene } \\
\hline \multicolumn{2}{|l|}{ Arbeiten im Haushalt } \\
\hline \multicolumn{2}{|l|}{ Soziale Aktiviäten } \\
\hline \multicolumn{2}{|l|}{ Vermeidung on Überanstrengung } \\
\hline \multicolumn{2}{|l|}{ Arbeit / Schule/Haushalt } \\
\hline Erholung & \\
\hline
\end{tabular}

Name Untersucher/in:

Unterschrift: 